\title{
Uwe Volkmann Wettbewerbsneutrale Parteienfinanzierung - ein Unding?
}

\begin{abstract}
Nachdem das Bundesverfassungsgericht sich mit Urreil vom 9.4. 199 I' von seiner bisherigen Judikatur mehr oder weniger übergangslos verabschiedet hat und das bisherige, von ihm selbst allerdings mitgeprägtc System der staatlichen Parteienfinanzierung als in weiten Teilen verfassungswidrig verworfen hat, ist durch das Sechste Gesetz zur Änderung des Parteiengesetzes und anderer Gesetze vom 28. r. $1994^{2}$ die fällige Neuregelung ins Werk gesetzt worden. Die Alimentierung der Parteien durch den Staat ruht nunmehr auf drei Säulen: der Wahlkampłkostenerstattung, der Aufstockung der Eigeneinnahmen der Parteien durch unmittelbare $\mathrm{Zu}$ schüsse und der steuerlichen Begünstigung von Parteispenden und Mitgliedsbeiträgen. Die Hoffnungen, daß sich diesc Säulen auch bci einer erneuten Prüfung in Karlsruhe als hinreichend standfest erweisen, sind freilich dünn. Die Neuregelung der Parteienfinanzierung ist, wic zuletzt verschiedentlich gezeigt ${ }^{3}$, in weiren Teilen mit den Vorgaben, die das Bundesverfassungsgericht seinerzeit aufgestellt hat, nicht vereinbar. Die Kritik offenbart freilich nicht nur, daß es sich bei der Partcienfinanzierung nach wie vor um eines der trüberen Kapitel bundesdcutscher Politik handelt, sondern zugleich das Dilemma, in das eine Gesetzgebung gcraten muß, die sich durch das höchste deutsche Gericht auf die Rolle eines Erfüllungsgehilfen bei der Umsetzung von Verfassungsrecht verwiesen sieht und nur noch versucht, die wenigen ihr durch die Karlsruher Spruchpraxis verblicbenen Handlungsspielräume auszunutzen. Sic soll daher in zwei Punkten aufgegriffen werden, weil dies Gelegenheir gibt, auf die zentrale Inkonsistenz der Entscheidung des Bundesverfassungsgericht und die Unmöglichkeit, auf ihrer Grundlage zu einem in sich stimmigen und mit sämtlichen Anforderungen der Verfassung vereinbaren Modell staatlicher Parteienfinanzierung zu gelangen, hinzuweisen. Mit der daraus resultierenden Notwendigkeit einer korrigiercnden Ungestaltung könnte sich indes überraschend die Chance eröffnen, das leidige Problem der staatlichen Partcienfinanzierung einer befriedigenden - und diesmal viellcicht abschließenden - Lösung zuzuführen.
\end{abstract}

\footnotetext{
1 BVCrfGE 85, 264 = NJW 1992, 2545.

2 BGBI. I, 142 .

3 Sendler, NJW 1994, 365; Drysch, NVwZ 1994, 218.
} 


\section{Die Begünstigung kleinerer Parteien und der Zwang zur Einbaltung der Obergrenze}

Die Neuregelung sieht unter anderem vor, daß Parteien jährlich für jede von ihnen bei einer Wahl erzielte Stimme bis zu einer Stimmenzahl von s Mio. einen Betrag von 1,30 DM, für jede darüber hinausgehende Stimme einen Betrag von I DM erhalten sollen; ergänzend zahlt der Staat auf jede von den Parteien eingenommene Mark aus Spenden und Beiträgen 0,50 DM hinzu (\$ I 8 III PartG). Um gleichwohl der vom Bundesverfassungsgericht geforderten nabsoluten Obergrenze", die sich aus dem Jahresmittelwert der den Parteien in den Jahren 1989 bis 1992 zugeflossenen öffentlichen Mittel ergeben sollte, Rechnung zu tragen, setzt $\$$ r 8 II PartG das Maximalvolumen staatlicher Zuwendungen - vorläufig' - auf 230 Mio. DM fest; wird dieser Betrag überschritten, erhält jede Partei nur den Beitrag, der ihrem Anteil an dieser Summe entspricht ( $\$ 19 V_{2}$ PartG). Die Einhaltung der an den selbst erwirtschafteten Mitteln einer Partei oricntierten "relativen Obergrenze soll durch die Kappungsvorschriften der $\$ \$ 18$ V I, I9 VI I PartG, nach denen staatliche Zuschüsse nur bis zur Höhe der jeweiligen Eigeneinnahmen vcrgeben werden dürfen, gewährleistet werden.

Mit diesem Inhalt ist die Neuregelung indes gleich in zweifacher Hinsicht mit dem vom BVerfG entwickelten Gebot der "Staatsfreiheit « der politischen Parteien - nach dem ein System staatlicher Zuwendungen die Parteien nicht der Notwendigkeit entheben darf, sich um die finanzielle Unterstützung durch die Bürger zu bemühen, und den jewciligen Erfolg einer Partei im Wettbewerb in hinreichendem Umfang berücksichtigen mu $\beta^{6}$ - nicht vereinbar. Zum einen wird die in den $\$ \$ 18 \mathrm{~V}, 19 \mathrm{VI}$ PartG enthaltene Kappungsgrenze wegen der vom Gesetzgeber vorgenommenen Kumulierung direkter Staatszuschüsse für alle Parteien schon bei eincr Wahlbeteiligung von $30 \%$ wirksam, so daß die staatliche Mittelvergabe aus sich heraus keinen Anreiz mehr für nennenswerte zusätzlichc Bemühungen um die Gunst von Wählern, Beitragszahlern und Spendern bietet; die beiden größten Parteien SPD und CDU könnten selbst Wählerverluste in Höhe von zweistelligen Prozentbeträgen verkraften, ohne daß sich dies auf der Einnahmenseite bemerkbar machen würde ${ }^{7}$ Zum anderen weist die in $₫$ r 8 III 2 PartG vorgeschene besondere Gewichtung der ersten s Mio. Stimmen im Rahmen der Wahlkampfkostenerstattung, die mit jeweils $x, 30 \mathrm{DM}$ - statt wie die darüber hinausgehenden Stimmen mit a DM - vergütet werden, fatale Parallelen zur Bestimmung des $₫$ i 8 VI PartG a. F." auf, die seinerzeit allen Parteien mit einem Stimmenanteil von über zwei Prozent einen gleichen, ebenfalls als Wahlkampfkostenerstattung deklarierten Grundbetrag verschaffen sollte". Die Gewährung eines solchen Sockels hatte das Bundesverfassungsgericht in seiner Entscheidung vom 9.4. 1992 mit der Begründung verworfen, sic sei nicht mehr hinreichend mit dem Erfolg einer Partei beim Wähler und bei der Einwerbung von Mitgliedsbeiträgen und Spenden verknüpft ${ }^{10}$.

4 Berucksichtigt werden jedoch nur Zurvendungen natürlicher Personen bis zu einem Betrag von $6000,-D M$

s Frhohungen sind nach Maßgabe von $\$ 18$ VI, VII PartG moglıch.

6 BVerfG NJW 1994,2545 (2546). Zu den Wandlungen, die der Begriff der Staatsfreiheit damit durchgemaclut hat, siehe Volkmann, ZRP 1992, $327 \mathrm{f}$.

7 Drysth, NVwZ 1994, 2216.

$8 \mathrm{BGBI} .1988 \mathrm{I}, 2615$.

9 Drysch, NVwZ 1994, 221 .

to BVerfG NJW $1994,2545(2548)$. Fur zulassig gehalten hatte das Gericht Icdiglich eine geringfugige Privilegierung der an der ; \%-Hurde scheiternden Parteien; das ist aber etwas anderes als die nunmehr vorgenommene gleichmȧßige Erhohung der Wahlkampfkostenerstattung fur die ersten s Mio. Stummen von ansonsten I DM auf 1,30 DM, Drysch a. a O. 
Auf die Einzelheiten sowie auf die zahlreichen weiteren Mängel der Neuregelung, auf die Drysch und in ähnlicher Weise auch Sendler" nachvollziehbar hingewiesen haben, soll hier indes nicht näher eingegangen werden. Im Rahmen dieses Beitrags interessiert nur der Effekt, den die Bestimmungen der $\$ S{ }_{1} 8$ III 2 und 19 VI PartG entfalten und der - wie dargelegt - einer Überprüfung durch das Bundesverfassungsgericht nach den von diesem selbst aufgestellten Maximen nicht standhalten könnte.

\section{Die Erfolgsabhängigkeit der Leistungsvergabe und die Neutralität des Staates gegenüber dem Parteienwettbewerb}

Die Besonderheit dieser beiden Regelungen liegt darin, daß sie in ihrem Zusammenspiel gerade jenen Maßstab aufweichen, der nach Auffassung des Bundesverfassungsgericht die Verteilung öffentlicher Leistungen künftig regulieren sollte: den von einer Partei im Wettbewerb mit anderen Parteien jeweils erzielten Erfolg. Die Anhebung der Wahlkampfkostenerstattung auf 1,30 DM im unteren Bereich bewirkt, als eine Art von Sockelbetrag, zunächst eine Besserstellung kleinerer Parteien, die dadurch im Ergebnis höhere Zuwendungen erhalten, als es ihrer - anhand des letzten Wahlergebnisses oder wie auch immer zu ermittelnden - Bedeutung entspräche; sie führt damit zu einer Leistungsverteilung, die die bestehenden Kräfteverhältnisse nicht exakt, sondern nur in einer abgeflacht proportionalen Form widerspiegelt. Zusätzlich relativiert wird die Verknüpfung von Mittelvergabe und (Wahl)Erfolg durch die gleichzeitige Kappung der Spitzenbeträge bei Erreichen der absoluten und relativen Obergrenze, weil sich jenseits dieser Grenze die von den Parteien errcichten Resultate nicht mehr in zusätzlichen Einnahmen niederschlagen können. In diesem, die Erfolgsabhängigkeit der Mittelverteilung relativierenden Effekt entspricht indes die Neuregelung den Anforderungen des Grundgesetzes eher als der erfolgsabhängige Finanzicrungsmodus, für den sich das Bundesverfassungsgericht im Urteil vom 9. 4. 1992 ausgesprochen hatte. Das ergibt sich, wenn man das Verteilungskriterium "Erfolg « auf den Prüfstand jenes Verfassungsprinzips stellt, dem man bislang die Antwort auf die Verteilungsfrage entnehmen zu können glaubte. Die Rede ist vom Grundsatz der Chancengleichheit der Parteien bzw. seiner Kehrseite, der Neutralität des Staates gegenüber dem Parteienwettbewerb.

Dieser Grundsatz folgt entgegen einer vielfach vertretenen Auffassung nicht aus Art. 3 I GG'2, sondern aus einem Agglomerat impliziter Verfassungssätze, die sämtlich auf die Funktion, die den Parteien für die politische Willensbildung zukommt, zurückverweisen's. Zu nennen wären:

- das Prinzip der Freiheit und Offenheit des politischen Prozesses ${ }^{14}$, das für den Staat die Vcrpflichtung zur Nichtidentifikation « mit bestimmten Meinungen oder einzelnen Gruppen begründer"s,

- die Zeitbegrenzung demokratischer Herrschaft, die jeder auf Dauer angelegten

it NJW 1994, 365. Unkritisch dagegen Hofmann, NJW 1994, 691.

12 Siehe etwa BVerfGE 7, 99 (108); 47, 199 (225); Julich, Chancengleichheit der Partesen, 1967, S. 74 ff.; Starck, in: von Mangoldt/Klein/Starck, Das Bonner Grundgesetz, Bd. I 1985, Art. 3 Rn. 31.

13 Die Eınzelheiten kónnen an dieser Stelle nicht dargelegt werden. Ausführlich Volkmann, Politische Parteien und offentliche Leistungen, 1993, 1 50 ff.

14 Dazu BVerfGE 20, 56 (97ff.); 44, I 25 (139); 73, 40 ( 84 f.); Hesse, Grundzige des Verfassungsrechts der Bundesrepublik Deutschland, i 8. Aufl. Rn, is9ff.

is Dazu Bockenfordc, VVDStRL 28, ss; Schlaich, Neutralitat als verfassungsrechtliches Prinzip, 1972, S. 236 ff. 
Verfestigung einer bestimmten Idee oder Gruppe im Staat entgegensteht ${ }^{16}$ und diesen tendenzicll auf Neutralität verpflichtet,

- das Mehrheitsprinzip, das nur auf der Grundlage gleicher rechtlicher Chancen aller Gruppen, zur Mehrheit zu werden, denkbar ist ${ }^{17}$ und vom Staat - als eine Umschreibung von Neutralität - die Respektierung dieser Chance verlangt,

- das Mehrparteicnsystem, in dessen Rahmen dic so entwickelten Grundsätze auf anderer Ebene - der des Parteien wettbewerbs - wiederkehren ${ }^{18}$,

- die Parteienfreiheit, die sich als Freiheit der Gründung, Betätigung und Erfolgsmöglichkeiten von Parteien zugleich als Garantie ihrer rechtlich gleichen Chancen erweist $^{19}$, die wiederum vom Staat - als Ausprägung von Neutralität - nicht geschmälert werden darf.

Wettbewerbsneutralität und Chancengleichheit verweisen demnach spiegelbildlich aufeinander; was sich für die Parteien als Anspruch auf Respektierung ihrer jeweiligen Entfaltungsmöglichkeiten darstellt, erscheint aus Sicht des Staates als Verpflichtung zur Wahrung strikter Unparteilichkeit. Zugleich wird offenbar, wie eine so hergeleitete Wettbewerbsneutralität mit der Rolle, die den Parteien im Demokratiemodell des Grundgesetzes obliegt, korrelicrt. Es sind die Parteien, denen im durch das Grundgesetz konstituierten freien, offenen und schon durch das Prinzip der Zeitbegrenzung demokratischer Herrschaft dynamischen politischen Prozeß, in dem Macht nach der Mchrheitsregel verteilt wird, ein zentraler Rang zukommt, und um dieses Ranges willen sind sie zugleich Subjekt und Objekt staatlicher Neutralitätspflichten. Wettbewerbsneutralität und Chancenglcichheit sichern und gewährleisten in diesem Sinne die Erfüllung der den Parteien obliegenden Funktionen, weshalb man sie als deren notwendiges Komplement bczeichnen kann: Nur in ciner von verzcrrenden Beeinflussungen des Staates freigehaltenen Konkurrenz können die Parteien den Bcitrag zur politischen Willensbildung entrichten, der ihnen durch Art. 21 GG auferlegt wird.

Wird Neutralität auf diese Weise als Komplement der Parteifunktion begriffen, dürfte sie einer ebenfalls aus der Funktion der Parteien im politischen Prozeß abgeleiteten "Staatsfreiheit", die vom Bundesverfassungsgericht mit Blick auf die Partei enfinanzierung ausdrücklich als Gebot, den politischen Prozeß und den Parteienwettbewerb offen zu halten, verstanden wird ${ }^{20}$, von vornherein nicht ins Gehege geraten. Das Gegenteil tritt jedoch ein, wenn sich der Umfang der Mittel, die eine bestimmte Partei erhält, nach dem von ihr jeweils errungenen Erfolg oder - was im Ergebnis keincn Unterschied macht - nach ihrer jeweiligen "Bedeutung " ${ }^{21}$ richten soll. Denn die damit verbundene Diffcrenzierung zwischen den Parteien enthält zum einen eine Bewertung, die als solche schon mit dem eigentlichen Wortsinn von Ncutralität in Widerspruch steht; wer neutral ist, darf diejenigen, denen gegenüber er Neutralität zu wahren hat, nicht nach bestimmten Kategorien als vorzugswürdig oder weniger vorzugswürdig beurteilen. Darüber hinaus läuft sie mit ihrer Anknüpfung an notwendig rückwärts gerichtete Kriterien wie den letzten Wahlerfolg oder die in der Vergangenheit erzielten Einnahmen einer Partei auf eine Zementicrung des Status quo hinaus, indem sie die früher erfolgreichen Parteien durch die Vergabe

16 Vgl. BVerfGE 44,125 (138f).

17 Heun, Das Mehrheitsprinzip in der Demokratie, 1983 , S. 194 ff.; Hesse (Fn. r 4 ), Rn. 143.

18 Auf das Mehrparteiensystem als Grundlage der Chancengleichheit greift auch das Bundesverfassungsgericht haiufig zuruick, Vgl. BVerfGE 44, $125(145) ; 73,40(65,89)$; siehe aus der Literatur Tsatsos/Morlok, Parteienrecht, 1981, S. 88; Grimm, in: Benda/Maihofer/Vogel, Handbuch des Verfassungsrechts der Bundesrepublik Deutschland, 1983, S. 343 f.

19 Hesse, VVDStRL :7, 36.

20 BVerfG NJW $1994,2545(2546)$.

21 So das in $\$ S$ I 2 PartG fur nicht-finanzielle Leistungen verzendete Differenzicrungskriterium. 
crhöhter Mittel in den Stand versetzt, die einmal errungenc Position auf Dauer zu bchaupten. $\mathrm{Da}$ auf diese Weise sowohl das Moment der Zeitbegrenzung demokratischer Herrschaft als auch die nicht zuletzt vom Bundesverfassungsgericht hervorgehobenc Freihcit und Offenheit des politischen Prozesses ${ }^{22}$-also Prinzipien, aus denen sich die Neutralität des Staates gegenüber den Parteien erst ergab - unterlaufen wird, versteht sich von selbst. In Mitleidenschaft gezogen wird dabei auch die den Parteien zuvörderst obliegende Funktion der Vermittlung zwischen Gesellschaft und Staat, auf deren Schutz es nach eigenem Bekunden gerade dem Bundesverfassungsgericht ankam. Parteien, die sich mangels Konkurrenz ihrer Stellung im politischen Prozeß sicher sein können, brauchen sich nicht mehr sonderlich um ihre Verwurzclung im Volk zu bemühen, sondern können jencs von ihrer gesellschaftlichen Basis abgehobene Dasein pflegen, dem eigentlich ein Riegel vorgeschoben werden sollte.

$\mathrm{Da}$ ß ein Einfrieren der Parteienkonkurrenz auf ihrem jetzigen Stand, von dem faktisch die aktuell einflußreichen Parteien einseitig profitieren, mit dem Grundsatz staatlicher Wettbewerbsneutralität nicht vereinbar sein kann, blieb allerdings auch dem Bundesverfassungsgericht nicht ganz verborgen. Weil bereits die Sperrklauseln des Wahlrechts das Aufkommen neuer Parteien erschweren und die Offenheit des Parteienwettbewerbes becinträchtigen, hielt es das Gericht immerhin für zulässig, den Parteien, die den Sprung in die Parlamente nicht geschafft haben, einen gewissen finanziellen Ausgleich zu gewähren ${ }^{23}$. Aufgehoben - zumindest teilweise - werden damit indes nicht dic Wirkungen eines gegen das Neutralitätsprinzip verstoßenden Finanzicrungsmodus, sondern die Wirkungen einer Wahlrechtsregelung, die das Bundesverfassungsgericht bislang stets für zulässig gehalten hatte und die von daher auch keiner - auch nicht auf dem Wege einer Finanzierungsregelung crfolgenden Korrcktur oder Milderung bedürfte ${ }^{24}$. Eine solche Korrektur kann umgekehrt den in einer bestimmten Ausgestaltung der Finanzierung liegenden Ncutralitätsverstoß nicht ausräumen.

Die an den jeweiligen Erfolg von Parteien gekoppelte Vergabe öffentlicher Leistungen bleibt nach alldem ein Problem für sich. Eine abgeflacht proportionale Leistungsverteilung, wie sie durch die neue Sockelbetragsregelung des $₫$ I 8 III 2 PartG bewirkt wird, dürfte jedenfalls einer aus der Funktion der politischen Parteien abgeleiteten Wettbewerbsneutralität weit eher entsprechen als das primär erfolgsorientierte Modell, das vom Bundesverfassungsgericht propagiert wird - wenn man sich nicht überhaupt auf den Standpunkt stellen will, daß aus den dargelegten Gründen nur eine strikt paritätische, also alle Parteien in gleicher Wcise begünstigende Subventionierung mit der Verfassung vereinbar ist $^{25}$. Dagegen spricht im wesentlichen nur das häufig vorgebrachte Argument, daß eine solche im Ergebnis zu einer Verzerrung der vorgefundenen Wettbewerbslage führe, Neutralität aber den Staat gerade auch zu deren Respektierung zwinge ${ }^{26}$; ein Argument, das freilich in dem Maße an Überzeugungskraft verliert, in dem die öffentliche Hand das andere an sie gerichtete Verfassungsgebot, also das Gebot einer wie auch immer zu definierenden Zurückhaltung bei der Vergabe öffentlicher Leistungen, ernstnehmen und den Umfang der Subventionierung der Parteien erheblich herunterschrauben würde ${ }^{27}$.

22 BVerfG NJW $1994,2545(2546)$

23 BVerfG NJW $1994,2545(2548)$.

24 Siehce zulctzt BVerfGE 82, 322.

2) Dafür etwa Seifert, Die politischen Parteien im Recht der Bundesrepublik Deutschland, s975, S. $147 \mathrm{ff}$., $308 \mathrm{ff}$; Lipphardt, Die Gleichheit der Parteien vor der öffentlichen Gewalt, 1975, S. 624 ff., 633 ff.; Kröger, in: Im Dienste Deutschlands und des Rechts, Festschrift fur W. Grewe, 1982, 507 ff.; von Munch, in: ders., Grundgesectkommentar, Bd. 2, 2. Aufl. 198 s, Arr. 21 Rn. 26 f., 29.

26 Vgl. BVerfGE 20, 56 (118); BVerWGE 47, 280 (289); Grimm (Fn. 18), S. 345 f.

27 Das Argument licgt zudem auf einer anderen Ebcne, siche Volkmann (Fn. 13), S. $226 \mathrm{ff}$. 
Das nunmehr etablierce System der Parteienfinanzierung wird demnach, will der Gesetzgeber nicht einen weiteren Rüffel des - in dieser Hinsicht freilich unberechenbaren - höchsten deutschen Gerichts riskieren, in Kürze erneut einer Revision unterzogen werden müssen. Dabei besteht einmal die - etwa von Drysch favorisierte ${ }^{28}$ - Möglichkeit, zu den Empfehlungen zurückzukehren, die die vom Bundespräsidenten im Anschluß an das Urteil des Bundesverfassungsgericht vom 9.4. 1992 einberufene Sachverständigenkommission seinerzeit ausgesprochen hatte ${ }^{29}$, die von den Parteien aber nach Kräften ignoriert wurden ${ }^{30}$; der Kommissionsbericht läuft immerhin noch auf die größtmögliche Annäherung an dic Instruktionen des Bundesverfassungsgericht hinaus. Angesichts der dargelegten Schwierigkeiten, diese Instruktionen mit dem Prinzip der Wettbewerbsneutralität zu harmonisieren, wäre es freilich auch denkbar, dic Entscheidung über das $\mathrm{Ob}$ und Wie staatlicher Parteienfinanzierung unmittelbar und ausschließlich wieder an diejenigen zu delegieren, die vom Grundgesetz zur Entscheidung politischer Fragen an vorderster Stelle berufen sind - an die Bürger, von denen alle Staatsgewalt an sich ausgehen soll. Diese wirken nach dem jetzt vom Gesetzgeber beschlossenen Modell der Parteienfinanzierung bei der Mittelverteilung zwar mit, aber entweder ohne ihr Zutun oder nicht in ihrer Gesamtheit. Soweit sich etwa der Umfang der staatlichen Zuwendungen nach der Wahlentscheidung richtet, ist die Stimmabgabe lediglich objektive Bedingung der Mittclzuweisung ( $\$$ I 8 III S. I Ziff. I u. 2, S. 2 PartG); der Wähler, der sich bei der Wahl für eine bestimmte Partei entscheidet, wendet dieser zwar damit gleichzeitig einen bestimmten Geldbetrag zu, aber er kann im Grunde nichts dafür - und es nützt ihm auch nichts, wenn er etwas dagegen hat ${ }^{31}$. Soweit schließlich der Staat Mitgliedsbeiträge und $S$ penden an die Parteien mittelbar oder unmittelbar bezuschußt $(\$ \$$ I 8 III \& Ziff. 3 PartG, Iob II, 34 g EStG), mag diese. Form der Subventionierung zwar dem Willen der Zuwender entsprechen. Diese befinden sich jedoch gegenüber der Gesamtheit der Wahlbevölkerung mehr oder weniger deutlich in der Minderheit; über die Verteilung staatlicher Mittel unter die Parteien entscheiden auf diese Weise nicht alle, sondern einige wenige.

Die Möglichkeit einer im strengen Wortsinn bürgernahen, weil umfassend bürgerbestimmten Parteienfinanzierung böte demgegenüber die schon oft vorgeschlagene, aber bislang immer verworfene Einführung des sogenannten Bürgerbeitrags in Form einer zusätzlichen "Finanzstimme«, mit der jeder Wähler anläßlich einer Wahl einer Parrei seiner Wahl cinen vorab ziffernmäßig festgelegten, aber vom Staat aufzubringenden Geldbetrag in der Größenordnung von etwa s-ro DM zuwenden könnte ${ }^{32}$. Diese Lösung verfügte im Unterschied zu den bisher behandelten über einc verfassungsrechtliche Rechtfertigung: aus Art. 20 II GG. Diese dürfe als einzige in der

28 Drysch, NVwZ 1993, $223 \mathrm{f}$.

29 Veroffenticht als BT-Drucks. I2/5774

$30 \mathrm{Vgl}$ dazu die Ausfuhrungen des Kommıssionsmitgliedes Sendler, YIW 1994, $36 \mathrm{~s}$

31 Unrichtig deshalb BVerfGE 24, 300 ( 344 f.) zur W/ahlkampfkrictinerstattung herkommlicher Pragung: die Bemessung des Erstattungsbetrages nach der bei eıner What errvichten Summenzahl trage wder Entscheidung der Aktivbirgerschaft uber dic Erstattung der Wahlkampfkustenerstattung und deren Umfang Rechnung a. Zahlreichen Wahlern durfte beı der Srimmabgabe nicht eınmal bekannt sein, daß sie damit 7.ugleich die Parteien finanzieren.

32 Der Vorschlap wurde in ahnlicher Form bereits im Berıcht der vom Bundesminister des Innern eingesetzten Parteienrechtskonmission, Rechtliche Neuordnung des Parteiwesens, 1957, S. 213 ff., unterbreitet und im Bericht z.ur Neuordnung der Parteienfinanzierung. Beilage zum Bundesanzeiger 1983, S. 217 f, aulgenommen; befurwortend seinerzeit von Arnım, Aktuelle Probleme der Parteienfinanzierung, S. 26. Fur den Burgerbeitrag hat sich neuerdings wieder Schutte, Burgernahe Parteienfinanzierung, 1993, stark gemache. 
Lage sein, die Anforderungen einer im strikten Wortsinn verstandenen Wettbewerbsneutralität einzuschränken ${ }^{33}$, mit der vorbehaltlos wohl nur noch das - gewiß auf keine allzu große Gegenliebe stoßende - Modell einer paritätischen Leistungsvertcilung bei gleichzeitiger erheblicher Reduzierung des Leistungsumfangs vereinbar wäre. Sie hätte zudem den Vorteil größtmöglicher Einfachheit und Klarheit, den man angesichts der bisherigen Erfahrungen - zu erinnern wäre etwa an die Regelung des Chancenausgleichs, die zu verstehen selbst ausgewiesene Experten Stunden brauchten ${ }^{34}$ und deren bloße Verlesung bei Kabarettveranstaltungen schon für Heiterkeit im Saale sorgtc - nicht gering schätzen sollte. Parteienfinanzierung würde bei Einführung des Bürgerbeitrags, vielleicht erstmals in einer langen Geschichte der Irrungen und Wirrungen, durchschaubar und transparent, und gesichert wäre zugleich der namentlich vom Bundesverfassungsgericht hervorgehobene Anreiz für die Parteien, sich um ihre Verwurzelung in der Gesellschaft hinreichend zu kümmern. Die Parteien müßten sich die Zustimmung der Bürger, von der sie und die Lebensfähigkeit des Parteienstaates abhängen, im doppelten Sinne des Wortes verdienen - ein vermurlich nicht allzu fürchterliches Los. 